\title{
WAYS TO INCREASE THE RANGE OF AN ELECTRIC VEHICLE
}

\author{
Donekal Pavan Kumar \\ Department of Mechanical Engineering \\ Hyderabad, Telangana, India
}

\begin{abstract}
This article is about the ways to increase the range of an EV. Electric vehicles are a collection of electronic and automobile parts, this article is mainly focused on altering the automobile parts to increase the range rather than electronic parts.
\end{abstract}

Keywords - Range, RPM, Aerodynamics.

\section{INTRODUCTION}

Electric vehicles are best known for non-pollutant vehicles and has bought revolutionary change in market. As electric vehicles run on battery, they consume less than $8 \%$ of power generated throughout the world and end up emitting no toxic gases into environment when compared to gasoline vehicles. In this paper, we will know various techniques to increase the range of an electric vehicle.

\section{WORKING OF AN ELECTRIC VEHICLE:}

The main parts of an electric vehicle are:

- Electric motor

- Battery

- Micro-controller

An electric vehicle has an electric motor which drives the wheels and powered by a battery pack \& need to be plugged in to a charging station or wall outlet to change.

There is a controller in a electric vehicle which senses the position of accelerator and adds more power to the motor which in-turn results in more speed.

\section{WAYS TO INCREASE RANGE}

Electric vehicle range can be increased not only by having a large battery which can produce power for a long time but also altering some of its components.

1. TYRES: Tyre's also have a part in increasing range of a vehicle. As power from the motor is directly received by tyre, change in tyre dimension or circumference may increase Range of electric vehicle. For ex. A circumference of a wheel is $\Pi$ times $D$. When the Diameter of the Tyre is altered, the distance for a single revolution varies. Thus range can be increased through some extent.

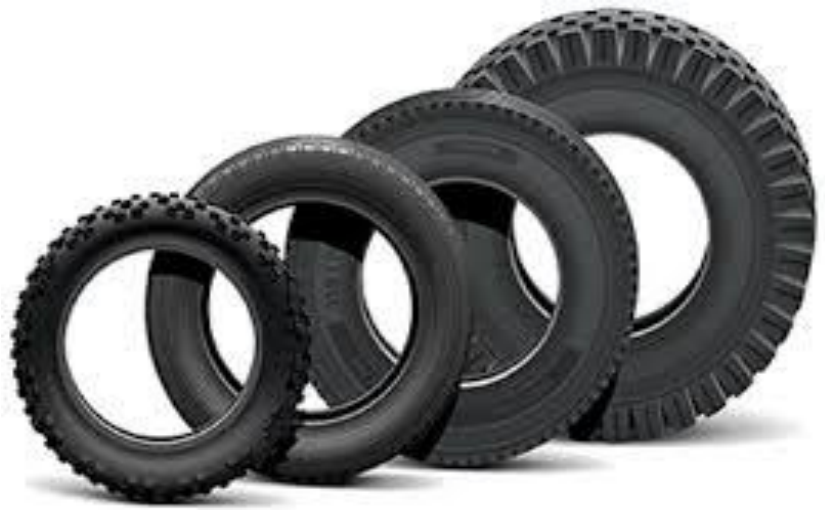

From the above image, the greater the diameter. The greater the circumference of the tyre.

2. MOTOR: Different types of motors are used in electric vehicles such as DC motors, Induction motors, Synchronous motors, Brush-less and Brushed Permanent magnet motors.

Motors play a major role in increasing range. The rotation of shaft in motor depends on the force exerted on it. By fleming's left hand rule where Force is directly proportional to Magnetic field strength in the motor \& Current passed. By passing same current and increasing the magnetic field inside the motor, the force on shaft is increased \& results in the increase of RPM, the more rpm the longer the vehicle travel.

The Magnetic field strength of the motor can be changed by Changing the number of turns of copper coil and by increasing the passage of current. The more number of turns, the increase in the magnetic field. The increase in the magnetic field, the more force on shaft. The more force on shaft, the more rpm, the more the rpm, the long the vehicle travel.

3. AERODYNAMICS: Aerodynamics has also a part in increasing the range of EV. The more the Drag resistance, the more power is consumed to come over. The Drag force can be reduced by decrease in the angle of window frame. The lesser the angle the lesser the air 
resistance I.e when moving at a higher speed the air impacts the window frame and flow off the window due to lesser in the angle, in case the angle of the window frame is more most likely a layer is formed on the window frame which slows down the vehicle and needs more fuel to come across it. Most of the sports car has less angle of window frame, that's the reason they can achieve more speed with less drag and spoilers are used to increase down-force. The more the down-force, the stable the vehicle therefore cannot effect the performance.

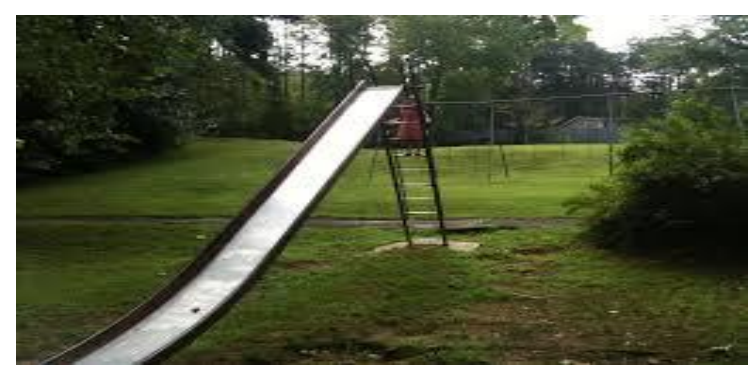

Figure-1

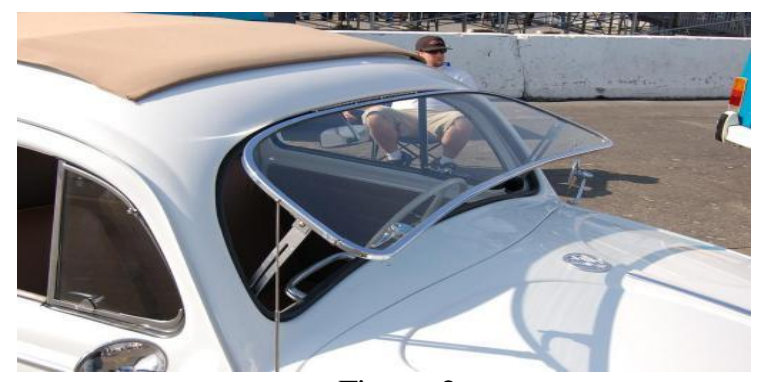

Figure-2

When window frame is designed at a less angle, it also guides the air to flow. When it is designed at a greater angle, there is no proper direction for the air to flow results in forming a layer on window frame and increases the resistance of flow.

4. REGENERATIVE BRAKING: Regenerative is an energy recovery system. the electric motor uses the vehicle's momentum to recover energy that would otherwise be lost to the brake discs as heat. Where as in conventional system, the kinetic energy is converted into heat energy due to friction in brakes. In electric motors, as soon the brakes are applied the motor acts as generators and the kinetic motion of wheels rotate the rotor and produces power which is used to charge the battery pack. However the partially recharged battery can add up few more extra miles and increase the range.

5. LONG LASTING: Do not charge your vehicle whenever it's in garage. Most of the batteries including lithium-ion will start self dis-charging when they have finished charging. You may be able to recover few more miles of range by charging it to full strength before you hit the road. This also increases battery's long-term health.

\section{CONCLUSION}

The are many more ways to increase the range of an electric vehicle. From the above, Magnetic field inside the motor \& Aerodynamics of the vehicle can be more effective to increase the range. Regenerative will be more effective to two wheeler as the battery pack of 2-wheeler is less when compared to 4wheeler. Battery performance can be estimated by modelling the Battery and circuits using MATLAB software to check the Temperature, SOC, SOH \& current.

\section{REFERENCE}

1. Chapter-4, Aerodynamics, Fundamentals of vehicle dynamics by THOMAS D GILLESPIE.

2. Darakhshan Anjum, 2018, Best car tyre's available, https://www.researchsnipers.com/best-car-tyresavailable-in-pakistan/

3. Hollywooddippa, 2011, Gallery- safari bug, https://www.thesamba.com/vw/gallery/pix/767121.jpg

4. Snapshot Take $\mathrm{r}$ R, Big slide at Chau ram park, https://mapio.net/pic/p-39383269/

5. Induction motors working by Team Learn Engineering, https://youtu.be/AQqyGNOP_30

6. Chapter 10, Tires, Fundamentals of vehicle dynamics by Thomas D Gillespie.

7. Regenerative Braking by Team Skill Lync, https://youtu.be/WA32wMdd28g

8. Jim Gorzelany, Boost EV,s Range, https://www.myev.com/research/buyers-sellersadvice/10-ways-to-boost-your-evs-range

9. Energy consumption prediction for electric vehicles based on real world data by Cedric de cauwer, Joeri van mierlo \& Thierry coosemans, 2015.

10. Wings \& Spoilers, Lift \& Drag by Team Donut Media, https://youtu.be/AXjiThF1LXU

11. Battery Modelling, Power electronics control design with simulink, Matlab.

12. Angular \& Linear speed of a circle/wheel Given rpm \& Radius Trig \& Precalculus by Team organic chemistry tutor, https://youtu.be/KM89GmqxFWg. 